\title{
A Multiple Access Scheme for Chaos-Based Digital Communication Systems Utilizing Transmitted Reference
}

\author{
Wai M. Tam, Francis C. M. Lau, Senior Member, IEEE, and Chi K. Tse, Senior Member, IEEE
}

\begin{abstract}
In this paper, a multiple access (MA) technique is proposed for chaos-based communication systems, in which chaotic reference signals are transmitted followed by the information-bearing signals. Chaotic reference signals modulated by a binary training sequence are sent periodically. The same chaotic signals are then modulated by the binary data and transmitted. To achieve MA, different chaotic signals and training sequences are assigned to different users. Two types of receivers are proposed. For the first one, an adaptive filter is employed which aims to reduce the inter-user interference. For the second receiver type, a simple inverse-and-average method is used in an attempt to recover the chaotic reference signal, which is then used to correlate with the information-bearing signals for determining the received symbols. The performance bounds of these two schemes are also derived. Finally, the bit error rates of the proposed system are simulated and compared.
\end{abstract}

Index Terms-Adaptive filter, bit error rates (BERs), chaos communications, multiple access (MA), noncoherent detection, Walsh codes.

\section{INTRODUCTION}

D IGITAL communications based on chaotic circuits were first proposed more than a decade ago [1], [2]. Since then, various modulation and demodulation schemes have been suggested and studied [3]-[8]. Compared with conventional communication schemes, chaos-based communication systems offer potential advantages such as ease of generation of broadband signal and high security. Recently, researchers have also begun looking into the MA capability of chaos-based communication systems which are inherently broadband. At the code level, spreading codes based on chaos have been applied to conventional direct-sequence code-division MA (DS-CDMA) systems [9]-[11]. Results show that using periodic quantized chaotic sequences as the spreading sequences can reduce the inter-user interference in an asynchronous DS-CDMA communication system. As a consequence, the capacity of the system can be enhanced. At the signal level, coherent and noncoherent MA systems have been proposed and analyzed [12]-[17]. In the coherent systems, exact replicas of the unmodulated chaotic

Manuscript received November 11, 2003; revised March 27, 2004. This work was supported in part by the Hong Kong Polytechnic University under the Young Professors Earmarked Research Grant 1-ZE03. This paper was recommended by Associate Editor O. Feely.

The authors are with the Department of Electronic and Information Engineering, The Hong Kong Polytechnic University, Hong Kong, (email: tamwm@eie.polyu.edu.hk; encmlau@ @olyu.edu.hk; encktse@ polyu.edu.hk).

Digital Object Identifier 10.1109/TCSI.2004.834497 carriers are assumed to be available at the receiver whereas no such requirement is needed for noncoherent systems.

Among the coherent MA chaos-based communication systems that have been proposed, the MA chaotic frequency modulation (CFM) and the MA chaos-shift-keying (CSK), respectively, extend the work of a single-user CFM system [4] and a single-user CSK system [3]. In the multiuser CFM environment [14], a base station will transmit a reference signal with chaotically varying frequency. Having received this reference signal, all mobile users in the system must first synchronize their own chaotic oscillators to it. Afterwards, each user can apply its own transformation to the synchronized chaotic waveform to generate its unique information-carrying CFM signal. To ensure that the reference CFM signal is free from interference, it is also required that the band in which the mobile units are transmitting the CFM signals is separated from the band used for "synchronization." In the coherent MA-CSK system [16], each transmitter generates two chaotic signals, one used to represent the binary symbol " +1 " and the other one for symbol " -1 ." At the receiver, the synchronized versions of the chaotic signals are assumed to be available and they correlate with the incoming signal. The symbol corresponding to the larger correlator output will then be decoded. Analytical techniques to studying the bit error performance of the MA-CSK system have also been derived [16]. Unfortunately, at the time of writing, robust chaos synchronization techniques for the practical noise levels concerned are still lacking, the study of coherent detection schemes remains only of academic interest and the performances of coherent systems are used mainly as benchmark indicators.

Noncoherent systems, which do not require the reproduction of the chaotic carriers at the receiving end, are more practical and improvements are still being made continually [5]-[7], [17]. In the single-user differential CSK (DCSK) system, each bit duration is divided into two equal slots. In the first slot, a reference chaotic signal is sent. Dependent upon the symbol being sent, the reference signal is either repeated or multiplied by the factor " -1 " and transmitted in the second slot. The extension of the DCSK scheme to a multiuser environment was first introduced by Kolumbán et al. [12]. For example, in a two-user environment, each bit period is divided into four time slots. For the first user, the reference signal is divided into two parts which are sent in the first and third time slots. Similarly, the information-bearing signal is also divided into two parts which are sent in the second and fourth time slots. To minimize the interference between the transmitted signals, the order of transmission is changed for the second user. The reference signal is 
sent in the first two slots while the information-bearing signal is transmitted in the third and fourth slots. By correlating different time slots of the incoming signal, different user can extract its own information with minimal interference from other users. Based on this MA-DCSK system, several enhancements have been made. For example, Jáko et al. replaced the chaotic signal with the CFM signal [13] and eliminated the autocorrelation estimation problem [17]. Lau et al. lowered the requirements on the switching circuits in the transmitter and the receiver by rearranging the the reference and information-bearing time slots of the users [15]. Also, by permutating the reference and information-bearing chaotic samples within each time slot, the similarity between the reference and information-bearing signals is removed, and data security is therefore enhanced [18].

Due to the small but finite interference between users, the performance of the MA DCSK and FM-DCSK systems degrades with the number of users. To eliminate the inter-user interference completely, Kolumbán et al. proposed the introduction of Walsh codes into the MA FM-DCSK system [17]. In their proposed scheme, two basis functions are used to represent the binary symbols for each user. Each bit duration is first divided into a number of time slots. To construct a pair of basis functions for each user, two Walsh codes are multiplied with a chaotic FM signal repeatedly. At a particular receiver, the chaotic FM signal in each time slot will multiply with the corresponding element in the Walsh code. Then, an averaging process is performed at the receiver to estimate the chaotic FM signal being sent. This is done for each of the two Walsh codes assigned to each user. Finally, each of the two estimated chaotic FM signals correlates with the received signal, and the symbol corresponding to the large correlator output will be decoded. If all the transmitted signals are synchronized at the bit level, the inter-user interference can be eliminated because of the orthogonal Walsh functions, and the performance is limited by the noise level only. To ensure the orthogonality between the basis functions of the users, longer Walsh codes may be required when the number of users increases. For example, in an $N$-user system, a total of $2 N$ basis functions are needed. Hence, each symbol period should be divided into $2^{L}$ time slots where $L$ is a positive integer and $2^{L} \geq 2 N$. Note that the number of time slots equals $2^{L}$ because this is the length of the Walsh codes. Compared with the single-user FM-DCSK scheme, a larger number of time slots and hence chaotic signals are now sent for each symbol. As a consequence, the bit rate will be lowered.

In this paper, a noncoherent MA scheme is proposed for chaos-based digital communication systems utilizing transmitted reference. In the proposed scheme, each user transmits reference chaotic signals, modulated by a training sequence, to the receiver periodically. Afterwards, the same reference signals are modulated by the data sequence to form the information-bearing signals. Also, users are differentiated by their reference chaotic signals and distinct training sequences. Two detection methods are studied. In the first method, an adaptive filter is employed. Based on the incoming training signals and the user's pre-assigned training sequence, the parameters of the adaptive filter are adjusted with an aim to minimizing the interference between users. After the training process has been completed, the adaptive filter is used to demodulate the

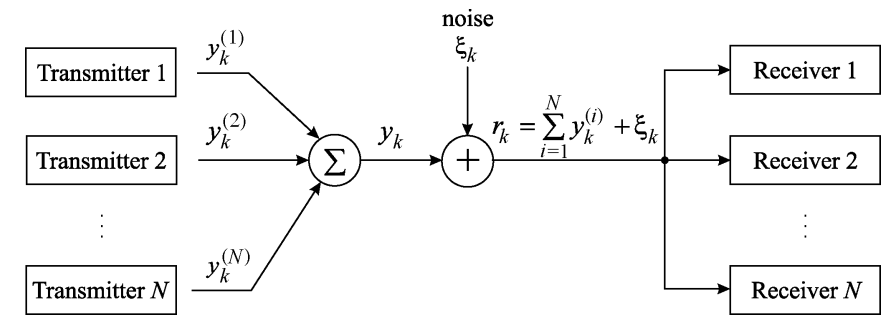

Fig. 1. MA chaos-based communication system under an additive white Gaussian noise environment.

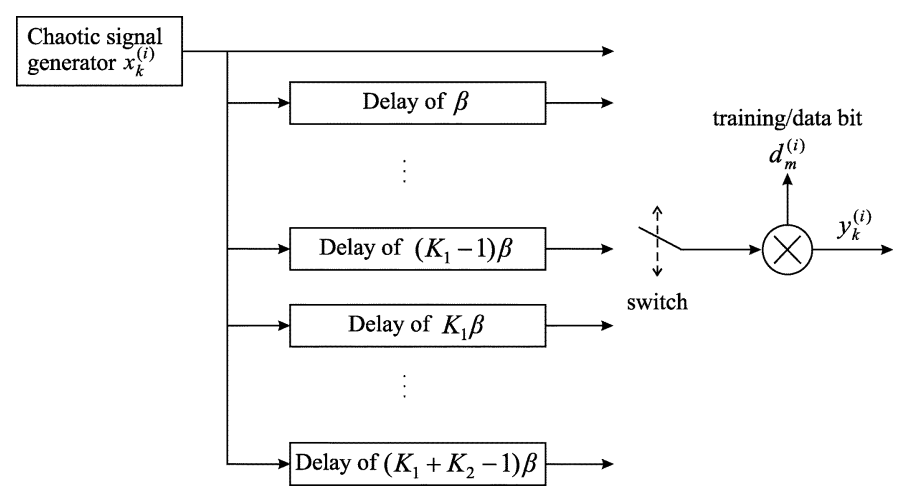

Fig. 2. Transmitter structure of the proposed MA chaos-based communication system.

information-bearing signals. In the second detection method, we first attempt to recover the reference chaotic signal. The incoming training signals in the time slots are multiplied by the user's pre-assigned training sequence. An averaging process is then performed to estimate the reference signal, which is used to correlate with the information-bearing signals for determining the received symbols. In a noiseless condition, clean reference chaotic signals can be recovered. In both detection schemes, each bit duration is divided into two time slots only. For simplicity, we only study the amplitude-modulated version of the system and compare the results with an earlier version of MA-DCSK system. However, the working principles of the transceivers can be easily adopted by chaotic FM signals. The only extra requirement would be to evaluate complex transmitted signals, complex tap coefficients, etc., at the transceivers instead of real ones.

The organization of the paper is as follows. We begin with the descriptions of the transmitter structure and the transmitted signal in Section II. In Section III, two receiver structures will be presented. The working principles of each receiver will be explained, together with a discussion on the performance bounds. Finally, we present the simulations results in Section IV.

\section{TRAnSmitTer Structure of NONCOHERENT MA ChaOs-Based Communication System}

Shown in Fig. 1 is an MA chaos-based communication system under a noisy environment. Here, we make use of the discrete-time baseband equivalent models of the transmitter and receiver to describe the proposed MA scheme [5], [8], [19]. Fig. 2 shows the transmitter structure of the $i$ th user. The transmitter consists of a chaos generator, a number of delay blocks, 


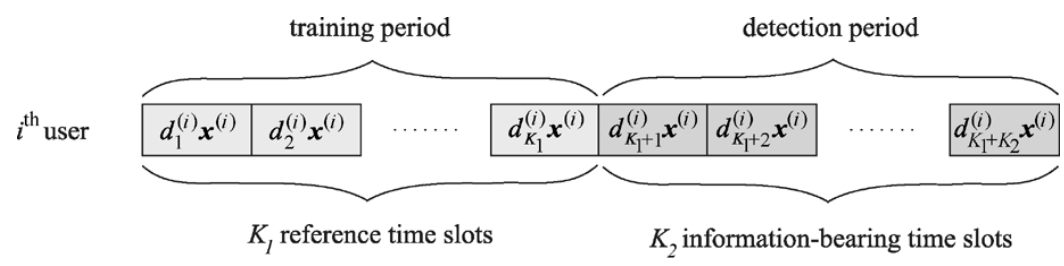

Fig. 3. Transmitted frame structure of the $i$ th user.

a switch and a multiplier. The transmitted signal is organized into frames which are further sub-divided into a number of time slots, as shown in Fig. 3. The first $K_{1}$ slots are used to send the reference chaotic samples for training, while the information-bearing chaotic samples are sent in the remaining $K_{2}$ slots. Without loss of generality, we consider the transmitted signal for a single frame period. At the output of the chaos generator, the chaotic signal is first biased before transmission such that its mean value is zero. This avoids transmission of the $\mathrm{dc}$ component which carries no useful information but increases transmission power. Suppose $\beta$ chaotic samples are sent within one slot. For each frame, the same $\beta$ chaotic samples will be used in each slot. We denote the chaotic samples used by the $i$ th user within this frame by $\left\{x_{k}^{(i)}: k=1,2, \ldots, \beta\right\}$. For algebraic brevity, we also define a chaotic-sample vector $\boldsymbol{x}^{(i)}$ as

$$
x^{(i)}=\left[\begin{array}{llll}
x_{1}^{(i)} & x_{2}^{(i)} & \cdots & x_{\beta}^{(i)}
\end{array}\right]^{T}
$$

where $T$ represents the matrix transposition.

Moreover, the chaotic-sample vector will be modulated by the training bits or the data bits before transmission. For the $m$ th time slot, the sample vector sent by the $i$ th user, denoted by $\boldsymbol{y}_{m}^{(i)}$, is given by

$$
\begin{aligned}
\boldsymbol{y}_{m}^{(i)} & =\left[\begin{array}{llll}
y_{(m-1) \beta+1}^{(i)} & y_{(m-1) \beta+2}^{(i)} & \cdots & y_{m \beta}^{(i)}
\end{array}\right]^{T} \\
& =d_{m}^{(i)} \boldsymbol{x}^{(i)}
\end{aligned}
$$

where $d_{m}^{(i)}$ equals " +1 " or " -1 ." When $1 \leq m \leq K_{1}, d_{m}^{(i)}$ represents the training bit, and when $K_{1}+1 \leq m \leq K_{1}+K_{2}, d_{m}^{(i)}$ denotes the information bit. In other words, if the training/data bit is " +1 ," the transmitted samples are the same as the chaotic samples. If the training/data bit equals " -1 ," the sign of the chaotic samples will be inverted and then transmitted. A typical frame structure for the $i$ th user is shown in Fig. 3.

Consider the $N$-user system shown in Fig. 1 . We assume that the frame sizes of all users are identical and the frames transmitted for all users are synchronized. Also, the number of slots per frame and the number of chaotic samples transmitted per slot are identical for all users. We further assume that $K_{1}=K_{2}=K$ such that on the average, two slots are required to send one data bit. Thus the average number of chaotic samples transmitted per bit (spreading factor) equals $2 \beta$. Assuming that all transmitters are switched on, the overall transmitted sample vector of the system, denoted by $\boldsymbol{y}_{m}$, is obtained by summing the outputs from all users, i.e.,

$$
\begin{aligned}
\boldsymbol{y}_{m} & =\left[\begin{array}{llll}
y_{(m-1) \beta+1} & y_{(m-1) \beta+2} & \cdots & y_{m \beta}
\end{array}\right]^{T} \\
& =\sum_{i=1}^{N} \boldsymbol{y}_{m}^{(i)} .
\end{aligned}
$$

\section{RECEIVER STRUCTURES OF NONCOHERENT MA ChaOS-BASEd COMMUNICATION System}

We make the usual assumption that the channel is additive white Gaussian. Thus, during the $m$ th time slot, the received sample vector, $\boldsymbol{r}_{m}$, is

$$
\begin{aligned}
\boldsymbol{r}_{m} & =\left[\begin{array}{llll}
r_{(m-1) \beta+1} & r_{(m-1) \beta+2} & \cdots & r_{m \beta}
\end{array}\right]^{T} \\
& =\boldsymbol{y}_{m}+\Phi_{m}
\end{aligned}
$$

where

$$
\Phi_{m}=\left[\begin{array}{llll}
\xi_{(m-1) \beta+1} & \xi_{(m-1) \beta+2} & \cdots & \xi_{m \beta}
\end{array}\right]^{T}
$$

and $\xi_{k}$ represents the $k$ th noise sample, the mean and variance (power-spectral density) of which are zero and $N_{0} / 2$, respectively. The first $K$ received sample vectors, i.e., $\left\{\boldsymbol{r}_{m}: m=\right.$ $1,2, \ldots, K\}$, are the training signals. Based on these training signals, the receiver needs to update its internal parameters before decoding the information-bearing vectors that follow. Two receiver structures will be investigated. The first one is based on an adaptive transversal filter (ATF) while the second one estimates the chaotic-sample vector from the training signals.

\section{A. Adaptive Transversal Filter}

1) Receiver Structure: Fig. 4 shows the structure of an ATF, which makes use of the first $K$ received sample vectors for updating the tap weights. The tap weights are set to zero for all users at the beginning of each training process. During the training period, the estimated training bit corresponding to the $m$ th slot of the $j$ th user, denoted by $\tilde{d}_{m}^{(j)}$, is first computed using

$$
\tilde{d}_{m}^{(j)}=\boldsymbol{r}_{m}^{T} w_{m-1}^{(j)}
$$

where

$$
\boldsymbol{w}_{m}^{(j)}=\left[\begin{array}{llll}
w_{m, 1}^{(j)} & w_{m, 2}^{(j)} & \cdots & w_{m, \beta}^{(j)}
\end{array}\right]^{T}
$$

is a vector containing the tap weights of the adaptive filter after the $m$ th $(m=1,2, \ldots, K)$ iteration (time slot). Then, the estimated training bit is compared with the desired symbol $d_{m}^{(j)}$. Based on the error between the desired symbol and the estimated symbol, i.e., $e_{m}^{(j)}=d_{m}^{(j)}-\tilde{d}_{m}^{(j)}$, the tap weights in each receiver are updated at the end of each time slot using the normalized least-mean-square (LMS) algorithm. The whole iterative process is summarized in the following steps [20]:

$$
\begin{aligned}
\boldsymbol{w}_{0}^{(j)} & =0 \\
e_{m}^{(j)} & =d_{m}^{(j)}-\tilde{d}_{m}^{(j)}=d_{m}^{(j)}-\boldsymbol{r}_{m}^{T} \boldsymbol{w}_{m-1}^{(j)} \\
\boldsymbol{w}_{m}^{(j)} & =\boldsymbol{w}_{m-1}^{(j)}+\frac{\mu}{a+\left\|\boldsymbol{r}_{m}\right\|^{2}} e_{m}^{(j)} \boldsymbol{r}_{m}, \\
& \quad 0<\mu<2, a \geq 0
\end{aligned}
$$




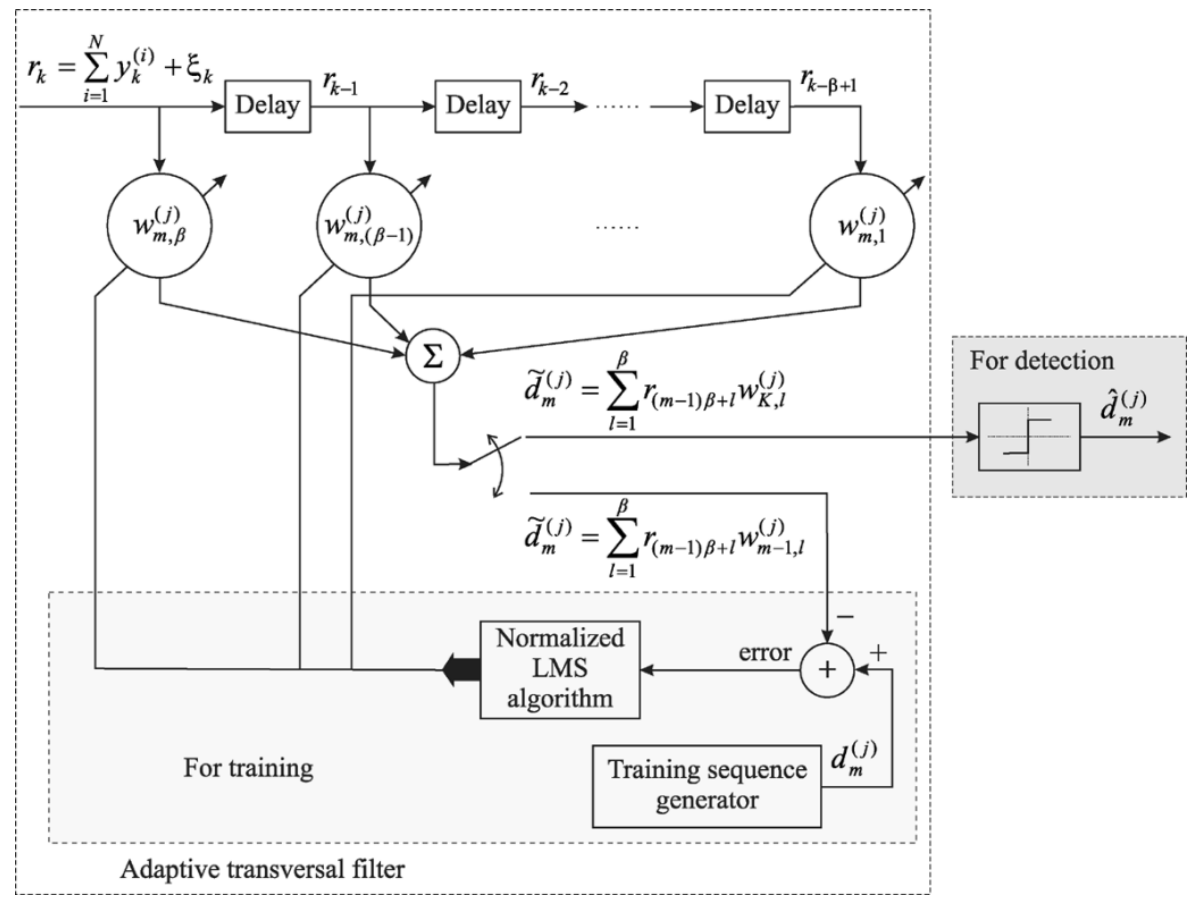

Fig. 4. Receiver based on an adaptive transversal filter.

where $\left\|\boldsymbol{r}_{m}\right\|$ is the Euclidean norm of the input vector $\boldsymbol{r}_{m}$ defined as

$$
\left\|\boldsymbol{r}_{m}\right\|=\sqrt{\sum_{l=1}^{\beta}\left[r_{(m-1) \beta+l}\right]^{2}}
$$

At the end of the training period, i.e., after $K$ iterations, we will obtain the tap-weight vector $\boldsymbol{w}_{K}$ which can then be used to estimate the data symbols embedded in the remaining time slots of the frame. The decoded data symbol, i.e., $\hat{d}_{m}^{(j)}, m=$ $K+1, K+2, \ldots, 2 K$, is then determined according to the following rule:

$\hat{d}_{m}^{(j)}=\left\{\begin{array}{cl}+1, & \text { if } \tilde{d}_{m}^{(j)}=\boldsymbol{r}_{m}^{T} \boldsymbol{w}_{K}^{(j)}>0, m=(K+1), \ldots, 2 K \\ -1, & \text { if } \tilde{d}_{m}^{(j)}=\boldsymbol{r}_{m}^{T} \boldsymbol{w}_{K}^{(j)} \leq 0, m=(K+1), \ldots, 2 K\end{array}\right.$

In other words, for the remaining $K$ time slots of each frame, if the estimated data symbol $\tilde{d}_{m}^{(j)}$ is larger than zero, then, " +1 " is detected. Otherwise, " -1 " is decoded.

2) Theoretical Performance Bound: Assume that the channel is noiseless during the training period. When the training period is long enough, the error between the desired data and the estimated data will approach zero for all users. Hence, we assume that the error $e_{m}^{(j)}$ equals zero for all $m>M$, where $M$ is a sufficiently large integer. In other words,

$$
e_{m}^{(j)}=0, \quad j=1,2, \ldots, N
$$

Based on (10), it can be further deduced that for all $m>M$

$$
\boldsymbol{w}_{m}^{(j)}=\boldsymbol{w}_{M}^{(j)}
$$

for all $j=1,2, \ldots, N$. Moreover, substituting (13) and (14) into (9), and making use of (1)to (4), we obtain

$$
\begin{aligned}
d_{m}^{(j)} & =\boldsymbol{r}_{m}^{T} \boldsymbol{w}_{M}^{(j)} \\
\Rightarrow d_{m}^{(j)} & =\boldsymbol{y}_{m}^{T} \boldsymbol{w}_{M}^{(j)} \\
\Rightarrow d_{m}^{(j)} & =\sum_{i=1}^{N}\left(d_{m}^{(i)}\left[\boldsymbol{x}^{(i)}\right]^{T} \boldsymbol{w}_{M}^{(j)}\right) \\
\Rightarrow & d_{m}^{(j)}=\sum_{i=1}^{N} d_{m}^{(i)}\left(\sum_{k=1}^{\boldsymbol{\beta}} x_{k}^{(i)} w_{M, k}^{(j)}\right), \quad j=1,2, \ldots, N \\
\Rightarrow & \sum_{k=1}^{\beta} x_{k}^{(i)} w_{M, k}^{(j)}=\delta_{i j}, \quad i, j=1,2, \ldots, N
\end{aligned}
$$

where $\delta_{i j}$ denotes the Kronecker's delta defined as

$$
\delta_{i j}= \begin{cases}1, & \text { when } i=j \\ 0, & \text { when } i \neq j .\end{cases}
$$

The equality in (15) shows that the correlation between the chaotic-sample vector of the $i$ th user and the tap-weight vector of the $j$ th transversal filter (receiver) equals zero and unity, respectively, for $i \neq j$ and $i=j$. That is to say, after the training process has been completed, the inter-user interference can be eliminated totally using the transversal filter, while the required signal can still be recognized and produces unity output at the transversal filter. Therefore, under such conditions, the cross-correlation estimation problem can also be minimized [17].

When the transversal filter is used to decode the informationbearing chaotic signal, which is now assumed to be corrupted by noise, the system behaves like a coherent single-user antipodal CSK system because the inter-user interference has been removed under a noiseless condition. The bit error rate (BER) 


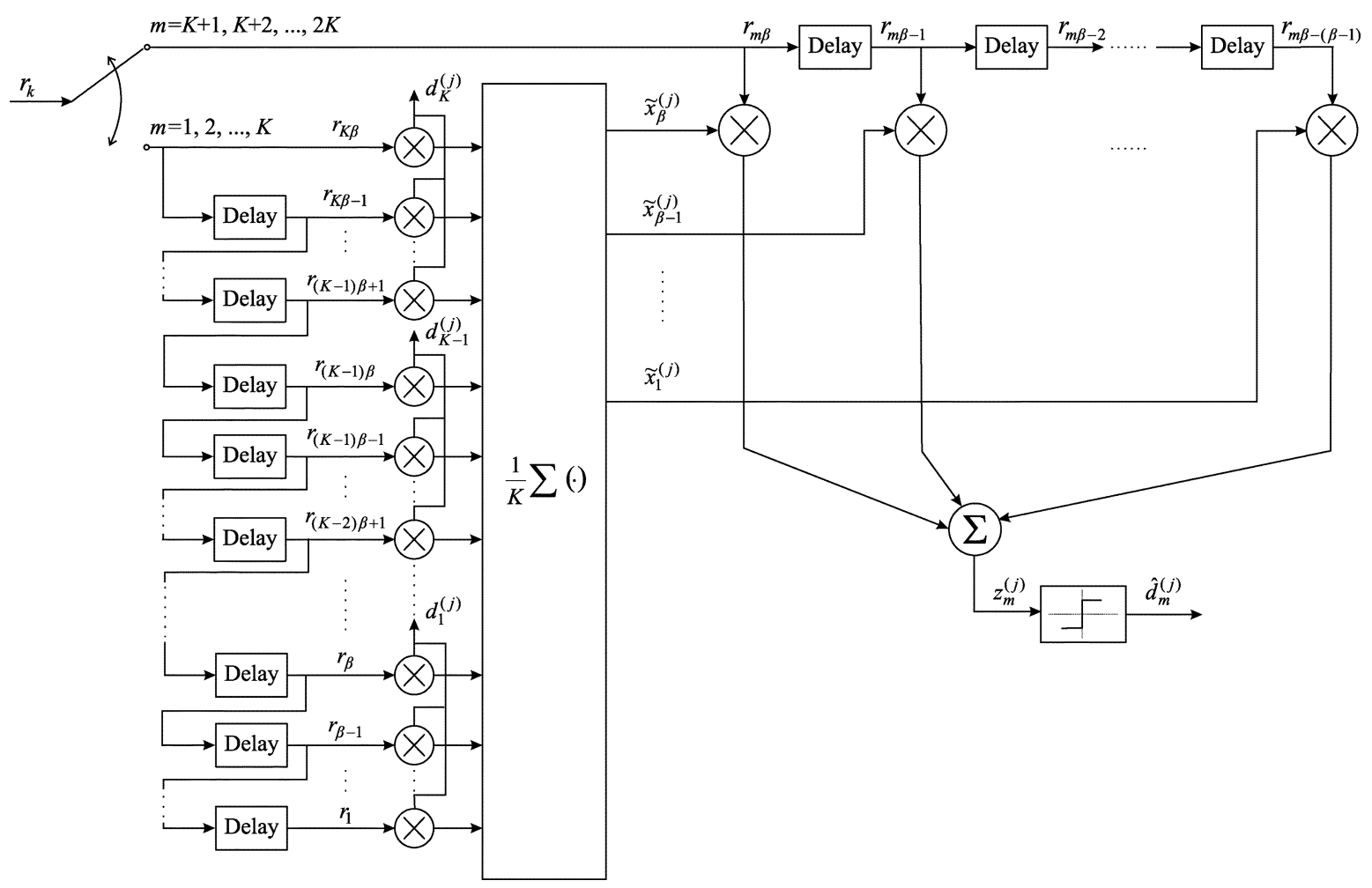

Fig. 5. Structure of the IA receiver.

of the aforementioned coherent CSK system is approximated by that of the conventional coherent binary phase-shift-keying (BPSK) communication scheme [21], and is given by

$$
\mathrm{BER}=\frac{1}{2} \operatorname{erfc}\left(\sqrt{\frac{\hat{E}_{b}}{N_{0}}}\right)
$$

where $\hat{E}_{b}$ denotes the energy of each bit in the demodulation process and erfc(.) is the complementary error function [19]. In the proposed noncoherent system under study, we assume that the number of training time slots and the number of information-bearing time slots are the same within each frame. As a consequence, if we denote the average bit energy of the $j$ th user by $E_{b}^{(j)}$, which equals

$$
E_{b}^{(j)}=2 \beta P_{s}^{(j)}
$$

where

$$
P_{s}^{(j)}=\mathrm{E}\left[\left(x_{k}^{(j)}\right)^{2}\right]
$$

denotes the average transmission power of the $j$ th user and E[.] represents the expectation operator, then $E_{b}^{(j)}$ will be twice as the bit energy spent in the demodulation process, i.e.,

$$
E_{b}^{(j)}=2 \hat{E}_{b}
$$

Substituting (20) into (17), the performance bound of the proposed receiver for the $j$ th user, denoted by $\operatorname{BER}^{(j)}$, equals

$$
\operatorname{BER}^{(j)}=\frac{1}{2} \operatorname{erfc}\left(\sqrt{\frac{E_{b}^{(j)}}{2 N_{0}}}\right) .
$$

In practice, the BER performance is expected to be worse than that given in (21) because the tap-weight vector cannot be estimated perfectly under the influence of noise and with a finite length of training sequence.

3) Choice of Training Sequences: In the proposed modulation scheme, the chaotic samples used to carry the data vary once every frame. At the beginning of each frame, the tap weights of the adaptive filter need to be reset and then updated so as to track the next set of chaotic samples. Hence, the training period should not be too long. To shorten the convergence time and to avoid getting the same tap weights for more than one user at the end of the training period, sets of orthogonal codes are assigned to the users as the training sequences.

Suppose $K=2^{n}$ where $n$ is a positive integer. We can construct $K$ orthogonal Walsh functions based on the Hadamard matrix $\boldsymbol{H}_{K}$, which can be generated using the following recursive procedure [19]:

$$
\begin{aligned}
\boldsymbol{H}_{1} & =[-1], \quad \text { for } n=0 \\
\boldsymbol{H}_{2} & =\left[\begin{array}{cc}
\boldsymbol{H}_{1} & \boldsymbol{H}_{1} \\
\boldsymbol{H}_{1} & -\boldsymbol{H}_{1}
\end{array}\right]=\left[\begin{array}{cc}
-1 & -1 \\
-1 & +1
\end{array}\right], \quad \text { for } n=1 \\
& \vdots \\
\boldsymbol{H}_{K} & =\left[\begin{array}{cc}
\boldsymbol{H}_{\frac{K}{2}} & \boldsymbol{H}_{\frac{K}{2}} \\
\boldsymbol{H}_{\frac{K}{2}} & -\boldsymbol{H}_{\frac{K}{2}}
\end{array}\right], \quad \text { for } n=\log _{2} K .
\end{aligned}
$$

Each row in the Hadamard matrix represents a Walsh code. Denote the $i$ th row of the $K \times K$ Hadamard matrix by $\boldsymbol{W}_{K \times K}^{(i)}$, $i=1,2, \ldots, K$. It is readily shown that the Walsh codes are orthogonal, i.e.,

$$
\boldsymbol{W}_{K \times K}^{(i)}\left[\boldsymbol{W}_{K \times K}^{(j)}\right]^{T}= \begin{cases}0, & \text { if } i \neq j \\ K, & \text { if } i=j\end{cases}
$$


The choice of $K$ depends on the number of users in the system. For example, $\boldsymbol{H}_{4}$ gives four orthogonal Walsh codes, and hence up to four users can be supported.

\section{B. Inverse-and-Average Receiver}

1) Receiver Structure: The structure of the inverse-and-average (IA) receiver is shown in Fig. 5. The roles of the receiver are to estimate the chaotic-sample vector of the required user during the training period, and to decode the information-bearing chaotic signal by correlating it with the estimated sample vector. To estimate the chaotic-sample vector, the modulation due to the training bit at the transmitter is first removed at the receiving end by multiplying the received signal in each time slot by the corresponding training bit again. Since the training bit equals \pm 1 , multiplying the chaotic-sample vector of the $j$ th user $\boldsymbol{x}^{(j)}$ twice (one at transmitter and another at receiver) by the same training bit produces no effect on the vector. Afterwards, the resultant vectors in all the training time slots are averaged to produce an estimation of the chaotic-sample vector which is represented by

$$
\tilde{x}^{(j)}=\left[\begin{array}{llll}
\tilde{x}_{1}^{(j)} & \tilde{x}_{2}^{(j)} & \cdots & \tilde{x}_{\beta}^{(j)}
\end{array}\right]^{T} .
$$

When the training process is finished, the chaotic signals in the information-bearing time slots will correlate with the estimated chaotic-sample vector. When the output of the $j$ th receiver, denoted by $z_{m}^{(j)}$, is larger than zero, a " +1 " is decoded. Otherwise, a " 1 " will be detected.

2) Theoretical Performance Bound: Using the same notations defined in Section II, for the $j$ th user, the recovered chaotic-sample vector is given by

$$
\begin{aligned}
\tilde{\boldsymbol{x}}^{(j)} & =\frac{1}{K} \sum_{m=1}^{K} d_{m}^{(j)} \boldsymbol{r}_{m} \\
& =\frac{1}{K} \sum_{m=1}^{K} d_{m}^{(j)}\left(\sum_{i=1}^{N} \boldsymbol{y}_{m}^{(i)}+\Phi_{m}\right) \\
& =\frac{1}{K} \sum_{m=1}^{K} d_{m}^{(j)}\left(\sum_{i=1}^{N} d_{m}^{(i)} \boldsymbol{x}^{(i)}+\Phi_{m}\right) \\
& =\boldsymbol{x}^{(j)}+\frac{1}{K} \sum_{m=1}^{K} \sum_{\substack{i=1 \\
i \neq j}}^{N} d_{m}^{(j)} d_{m}^{(i)} \boldsymbol{x}^{(i)}+\frac{1}{K} \sum_{m=1}^{K} d_{m}^{(j)} \Phi_{m} .
\end{aligned}
$$

In (27), the first term is the required chaotic-sample vector. Both the second and third terms are unwanted signals and should be removed. The third term, being derived from the noise, cannot be eliminated. The second term, however, is introduced by the transmitted signals of other users and can be removed by assigning appropriate training sequences to the users. Re-arranging the summation signs in the second term in (27), we have

$$
\frac{1}{K} \sum_{m=1}^{K} \sum_{\substack{i=1 \\ i \neq j}}^{N} d_{m}^{(j)} d_{m}^{(i)} \boldsymbol{x}^{(i)}=\frac{1}{K} \sum_{\substack{i=1 \\ i \neq j}}^{N} \boldsymbol{x}^{(i)}\left(\sum_{m=1}^{K} d_{m}^{(j)} d_{m}^{(i)}\right) .
$$

From (28), it can be observed that the effect of other users can be removed by assigning training sequences $\left\{d_{m}^{(i)}: i=\right.$ $1,2, \ldots, N\}$ such that

$$
\sum_{m=1}^{K} d_{m}^{(j)} d_{m}^{(i)}=0
$$

for $i \neq j$. This can be easily accomplished by assigning Walsh codes as the training sequences, as in Section III-A-3. With such an arrangement, the estimated chaotic-sample vector becomes

$$
\tilde{\boldsymbol{x}}^{(j)}=\boldsymbol{x}^{(j)}+\frac{1}{K} \sum_{m=1}^{K} d_{m}^{(j)} \Phi_{m} .
$$

The estimated chaotic-sample vector $\tilde{\boldsymbol{x}}^{(j)}$ will then correlate with the chaotic signals transmitted in the information-bearing time slots. Denote the input to the detector by $z_{m}^{(j)}$ for the $m$ th time slot of the $j$ th user. Then,

$$
z_{m}^{(j)}=\left[\tilde{\boldsymbol{x}}^{(j)}\right]^{T} \boldsymbol{r}_{m}
$$

The received symbol, denoted by $\hat{d}_{m}^{(j)}$, is decoded according to the following rule:

$$
\hat{d}_{m}^{(j)}= \begin{cases}+1, & \text { if } z_{m}^{(j)}>0, \\ -1, & \text { if } z_{m}^{(j)} \leq 0, \quad m=(K+1), \ldots, 2 K\end{cases}
$$

To estimate the performance bound, we further assume that the number of reference time slots $K$ is large enough such that the term due to noise can be ignored in (30). Therefore, (30) is simplified to

$$
\tilde{x}^{(j)} \approx x^{(j)}
$$

implying that a clean chaotic-sample vector can be recovered at the receiver. Under such circumstances, during the decoding of the information-bearing time slots, the communication system is equivalent to a multi-user antipodal coherent CSK system. Therefore, the performance of the IA receiver is bounded by that of the multi-user antipodal coherent CSK system, which will be found by computer simulations in the following section.

Comparing the complexity of the two proposed receivers in this section, it can be seen that the receiver based on an ATF has a slightly more complicated structure (mainly because of the LMS algorithm) over the IA receiver.

\section{RESUlTS AND DISCUSSION}

In our simulations, all users use the same map, each with a different initial condition, to generate the chaotic samples. The form of the map is given by

$$
x_{k+1}=4 x_{k}^{3}-3 x_{k} .
$$

With this map, the average transmission power of each user $P_{s}$ is readily shown equal to 0.5 [8]. It can be substituted into (18) in computing the performance bound equation of the ATF receiver, as given in (21). Also, for the ATF receivers, the parameters $\mu$ 


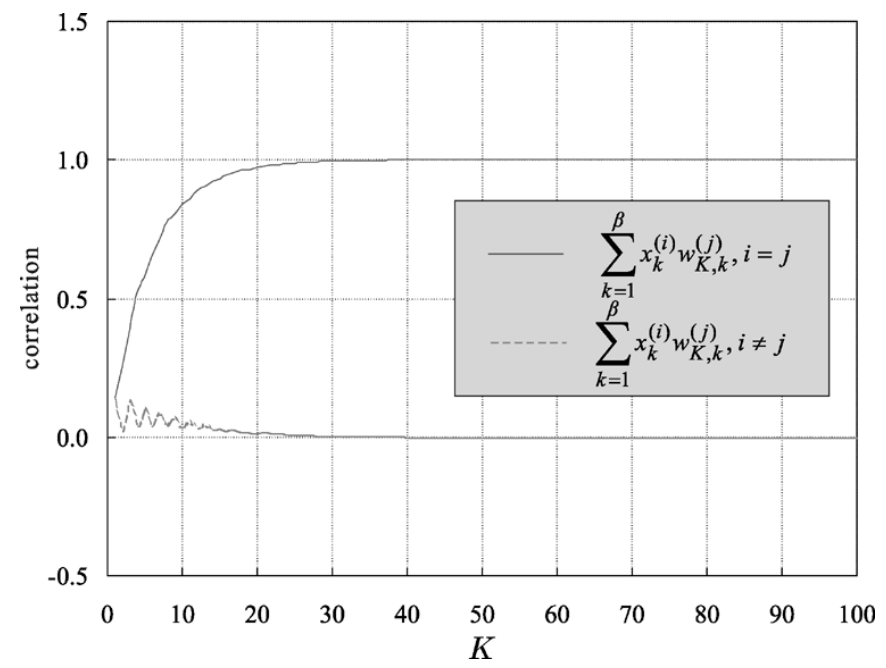

Fig. 6. Correlation of $\boldsymbol{x}^{(i)}$ and $\boldsymbol{w}_{K}^{(j)}$ versus $K$ for an ATF receiver under a noiseless environment. Number of users $=4$. Spreading factor $=200$.

and $a$ in the LMS algorithm are set to 0.5 and $10^{-20}$, respectively. The BERs of the proposed system are then simulated to reveal the effects of varying the following parameters:

- bit-energy-to-noise-power-spectral-density ratio $\left(E_{b} / N_{0}\right)$ which is given by $2 \beta P_{s} / N_{0}$;

- length of training sequence $K$;

- number of users $N$; and

- spreading factor $2 \beta$.

For comparison, we will show the BERs of the typical timedelay-based multiple-access approach, such as the MA-DCSK system proposed by Kolumbán [12] and studied by Lau et al. [15]. The performance bounds of the ATF receiver (21) and IA receiver (by simulations) will also be plotted as references.

First, we evaluate how the length of the training sequence affects the training result of the ATF receiver under a noiseless environment. Fig. 6 plots the correlation of $\boldsymbol{x}^{(i)}$ and $\boldsymbol{w}_{K}^{(j)}$, i.e., $\sum_{k=1}^{\beta} x_{k}^{(i)} w_{K, k}^{(j)}$, versus $K$. As shown by the curves, when the length of the training sequence increases, the correlation value approaches unity and zero, respectively, for $i=j$ and $i \neq$ $j$. The results verify that the ATF receiver has the capability of recognizing the required signal and removing the inter-user interference after the training process.

In the next set of simulations, we study a single-user system and vary the length of the training sequence. The corresponding BER curves are plotted in Fig. 7. For the case with $K=1$, the structure of the transmitted signal is the same as that of a singleuser DCSK system [3], the BER performance of which is also shown in the figure for comparison. Also, the structure of the IA receiver is the same as the noncoherent DCSK receiver when $K=1$. From the results, it can be observed that for $K=1$, the ATF receiver achieves similar BERs as the DCSK scheme (and consequently, the IA receiver) in a single-user environment. For $K=4,8$ and 16 , the ATF and the IA receivers have similar performance. Also, when the length of the training sequence $K$ increases, the BERs of both types of receivers have improved because a larger number of training signals can reduce the effect of noise during the training process.

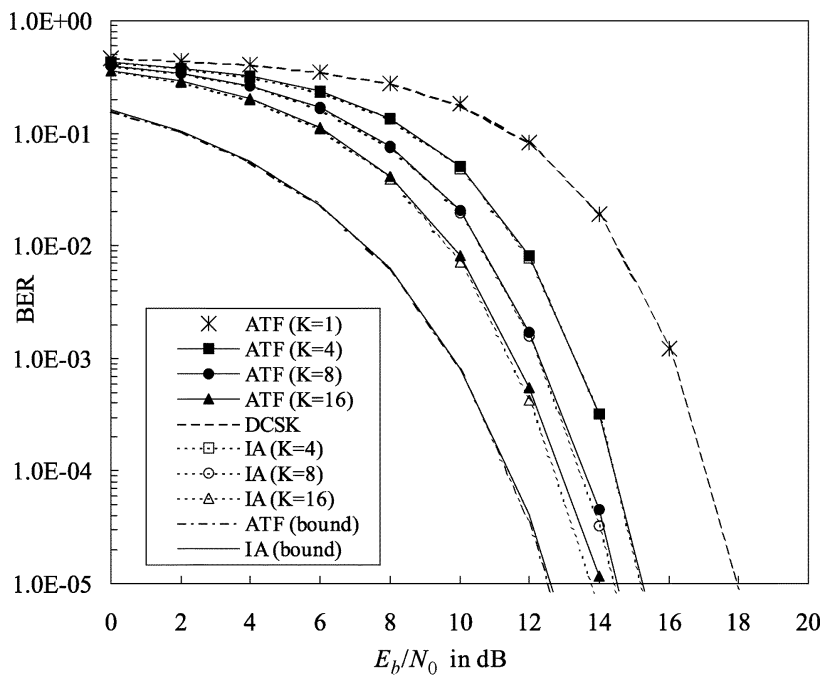

Fig. 7. BER versus $E_{b} / N_{0}$ for the proposed chaos-based communication system with one user. $K=1,4,8$ and 16. Spreading factor is 200 .

Fig. 8 plots the BERs versus $E_{b} / N_{0}$ for a four-user system. The length of the training sequence $K$ is equal to 4,8 , and 16 . Spreading factors of 20, 40, 100, and 200 are employed. The BER curve for the MA-DCSK system is also shown for comparison [22]. Here, we see clearly that the ATF receiver significantly outperforms the IA receiver and the MA-DCSK scheme in all cases. This is because the ATF receiver can effectively reduce the inter-user interference whereas the IA receiver and the MA-DCSK scheme do not possess such capability. Comparing the IA receiver and the MA-DCSK scheme, the IA receiver can always estimate a "cleaner" reference chaotic signal used for demodulation. Thus, its BER performance is better than that of the MA-DCSK scheme. In Figs. 8(a) and (b), it can also be observed that when the noise power is reduced to a very low level (say, $E_{b} / N_{0}$ over $16 \mathrm{~dB}$ ), the BERs do not improve very much. This is because the BER performance is now limited by the autocorrelation and cross-correlation estimation problems [17]. By increasing the spreading factor, the results in Figs. 8(c) and (d) indicate that the estimation problems become less significant and the BER continues to improve with lower noise level. For the ATF receiver, it should be noted that the cross-correlation estimation problem can also be alleviated by increasing the length of training sequence because a well-trained ATF receiver can effectively eliminate the inter-user interference.

Fig. 9 plots the BER curves versus $E_{b} / N_{0}$ for 2-, 4- and 8 -user systems. The length of the training sequence equals 16 and the spreading factor used is 200 . The corresponding curves for the MA-DCSK system are also shown in the figures. It can be observed that the BER increases (degrades) with the number of users, the reason being an increase in inter-user interference. Compared to that of the IA receiver and the MA-DCSK scheme, the degradation of the ATF receiver is smaller because the ATF receiver has the capability of reducing the effects of inter-user interference effectively. Thus, the ATF receiver can achieve a better BER compared with the IA receiver and the MA-DCSK scheme. For a large number of users, the performance discrepancy between the ATF and IA receivers is large. This is because the ATF receiver can alleviate the inter-user interference 


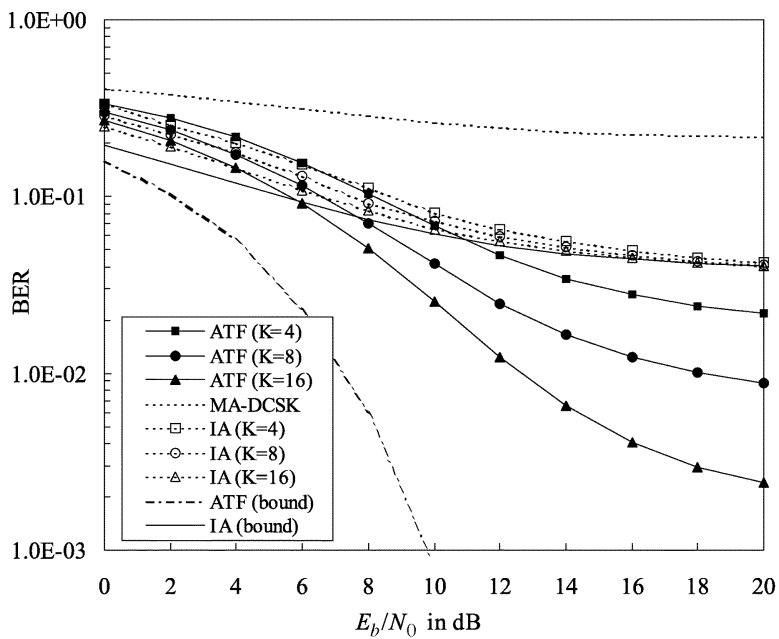

(a)

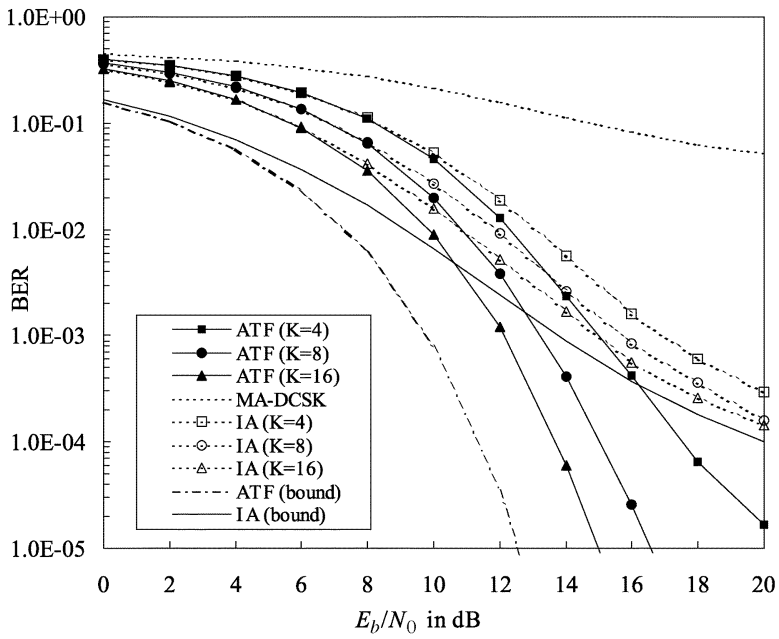

(c)

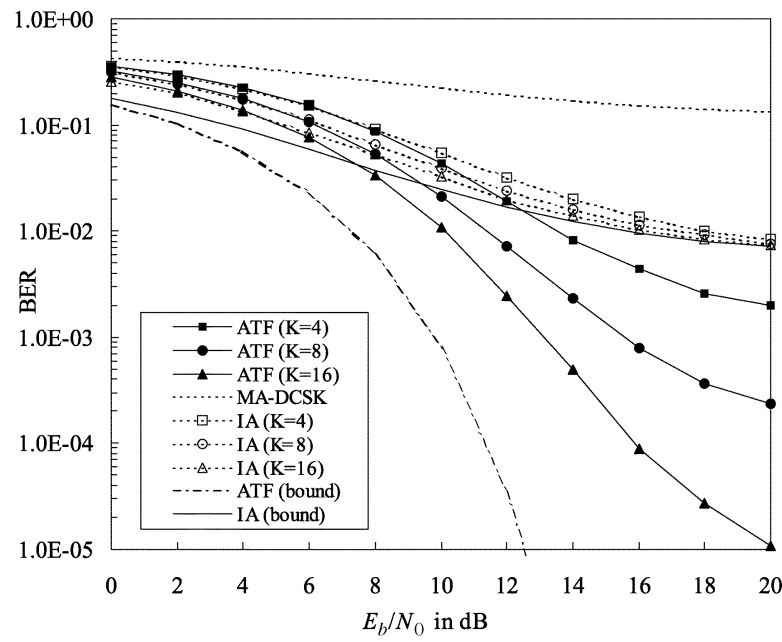

(b)

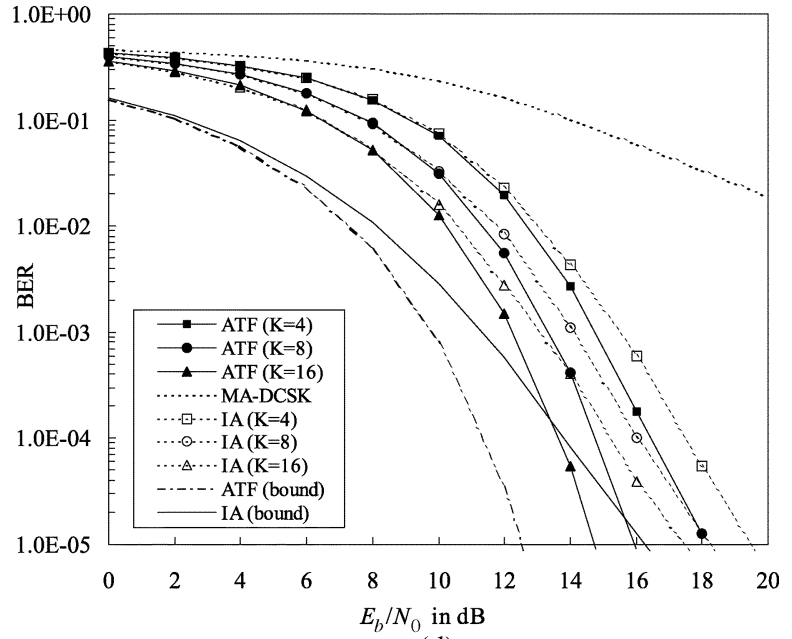

(d)

Fig. 8. BER versus $E_{b} / N_{0}$ for the proposed chaos-based communication system with four users. Spreading factor is (a) 20, (b) 40, (c) 100 , and (d) 200 .

whereas the IA receiver aims only to recover a clean chaotic reference signal from the training signal. The MA-DCSK scheme has the worst BER performance because both of its reference and information-bearing signals are suffering from noise and inter-user interference.

In Fig. 10, we plot the BERs against the spreading factor in 2-, 4- and 8-user systems with $K=8$ and 16 for the ATF receiver. For the IA receiver, the BER results are not presented because the trends are very similar to those of the ATF receiver. From Fig. 10, it is shown that as the spreading factor increases, the BER improves initially, but reaches an optimal value at a certain spreading factor beyond which performance starts to degrade. The decrease in the BER is due to the reduced effect of the auto-correlation and cross-correlation estimation problems, while the increase in the BER is caused by the large noise level.

Finally, we investigate the proposed noncoherent MA system under a noiseless environment. In Fig. 11, the BERs of the ATF and IA receivers are plotted against the number of users for different spreading factors. As expected, the BERs increase with the number of users because of the increase in inter-user interference. Also, the BERs reduce with the spreading factor because the chaotic signals sent by different users can be more easily dis- tinguished from one another when the spreading factor is larger. Moreover, it can be observed that the ATF receiver performs better than the IA receiver in all cases. In Fig. 12, the BERs are plotted against the number of users for different lengths of the training sequence. The performance of both ATF and IA receivers degrades with the number of users. For the ATF receiver, the BER reduces as $K$ increases because a longer training sequence enables the ATF receiver to remove the inter-user interference more effectively. Under a noiseless condition, the performance of the IA receiver is independent of $K$ because a clean reference chaotic signal can always be recovered. Therefore, only one curve is shown for the IA receiver. As explained earlier, since the IA receiver cannot eliminate the inter-user interference, its performance is always worse than that of the ATF receiver.

\section{CONCLUSION}

In this paper, we have proposed a noncoherent MA chaosbased communication system utilizing transmitted reference. The transmission scheme is simple and easy to implement. Essentially, a series of training chaotic signals are sent to train the receivers at the receiving side. Two types of receivers have been proposed and studied. The first one is based on an ATF. By using the normalized least-mean-square algorithm to update the 


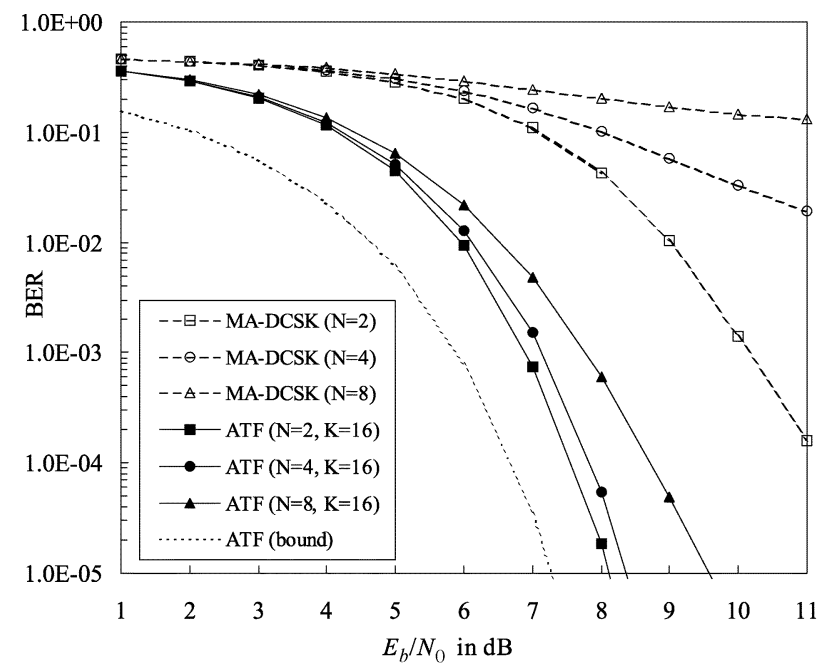

(a)

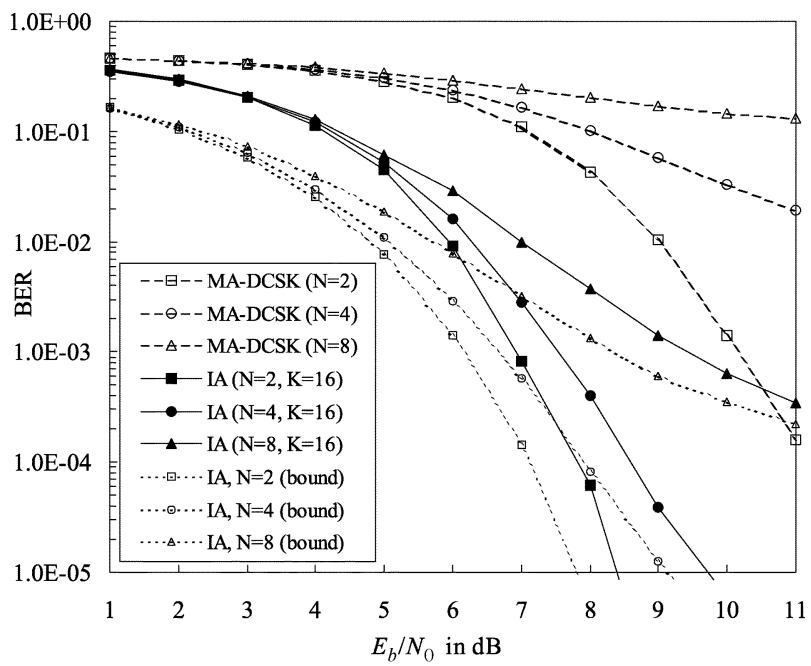

(b)

Fig. 9. BER versus $E_{b} / N_{0}$ for the proposed chaos-based communication system with 2, 4, and 8 users. $K=16$ and spreading factor is 200 . (a) Comparison between ATF receiver and MA-DCSK scheme. (b) Comparison between IA receiver and MA-DCSK scheme.

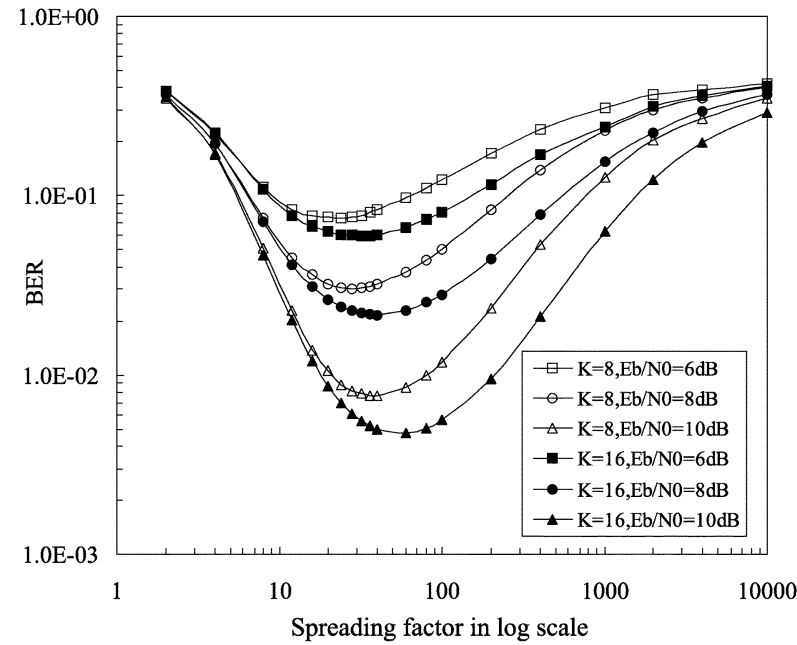

(a)

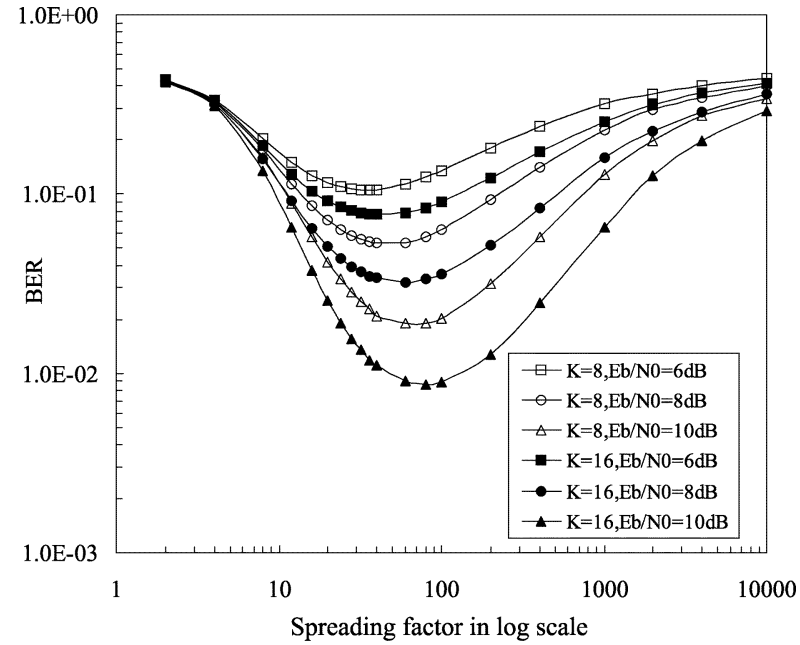

(b)

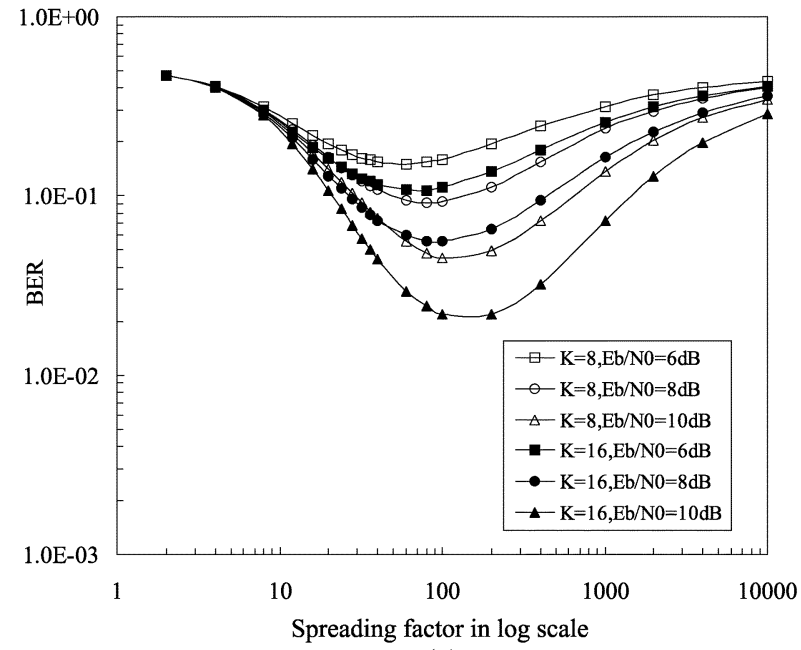

(c)

Fig. 10. BER versus spreading factor for the proposed chaos-based communication system employing ATF receivers. Number of users is (a) $N=2$, (b) $N=4$, and (c) $N=8$. 


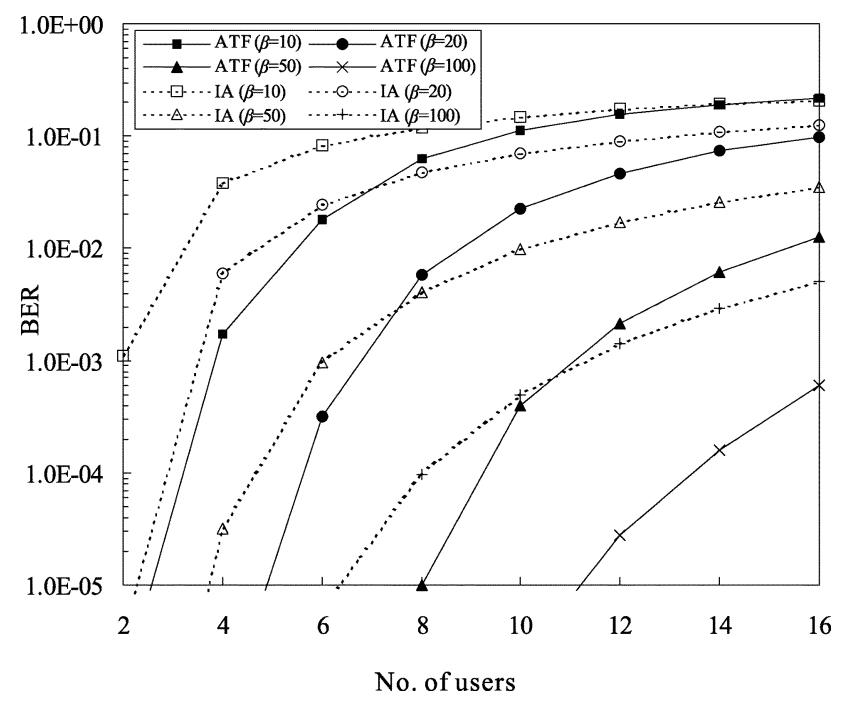

Fig. 11. BER versus number of users for the proposed chaos-based communication system for different values of spreading factor in a noiseless environment. $K=16$.

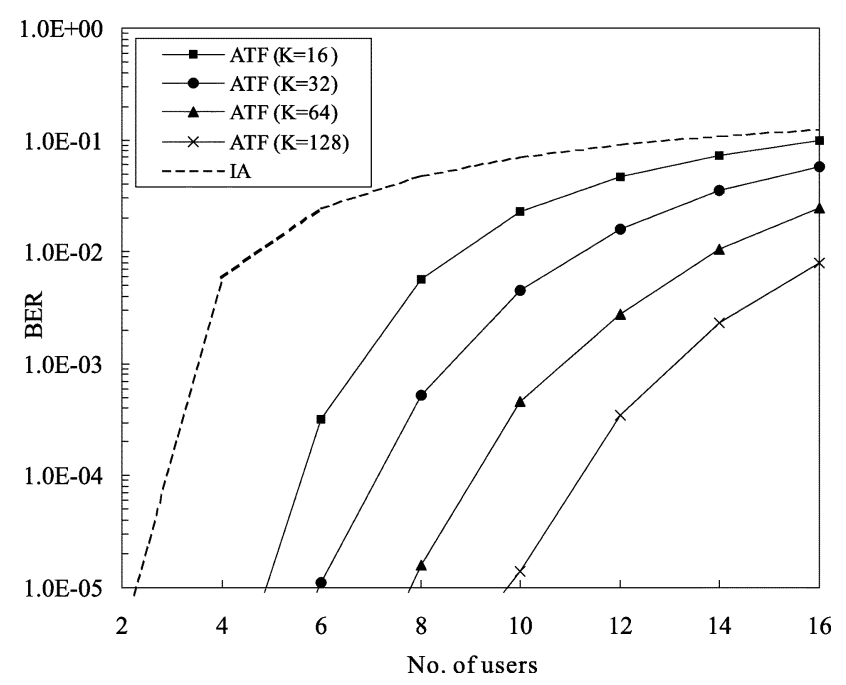

Fig. 12. BER versus number of users for the proposed chaos-ebased communication system for different values of $K$ in a noiseless environment. Spreading factor is 40 .

tap weights of the filter, the mean-square-error between the incoming training symbols and the expected symbols is reduced, which in turn mitigates the interference between users when decoding the information-bearing signal. The second type of receiver, namely the IA receiver, aims at recovering clean reference chaotic signals by multiplying the signals in the reference time slots by the corresponding training bits, followed by averaging. Comparing the complexity of the two receivers, the ATF receiver has a slightly more complicated training algorithm over the IA receiver. However, results show that the ATF receiver outperforms the IA receiver and other time-delay-based multiple-access system. Although an amplitude-modulated chaotic carrier is studied in this paper, the modulation/demodulation schemes described here can be easily extended to frequencymodulated chaotic carriers by using complex transmitted signals and complex tap coefficients in the transceivers instead of real values. Also, because of the adaptive nature of the ATF receiver, the proposed system should offer an improved performance over other MA systems in a time-varying channel.

\section{REFERENCES}

[1] U. Parlitz, L. O. Chua, L. Kocarev, K. S. Halle, and A. Shang, "Transmission of digital signals by chaotic synchronization," Int. J. Bifurc. Chaos, vol. 2, pp. 973-977, 1992.

[2] H. Dedieu, M. P. Kennedy, and M. Hasler, "Chaos shift keying: modulation and demodulation of a chaotic carrier using self-synchronizing Chua's circuit," IEEE Trans. Circuits Syst. II, vol. 40, pp. 634-642, Oct 1993.

[3] G. Kolumbán, M. P. Kennedy, and L. O. Chua, "The role of synchronization in digital communications using chaos-part II: chaotic modulation and chaotic synchronization," IEEE Trans. Circuits Syst. I, vol. 45, pp. 1129-1140, Nov. 1998.

[4] A. R. Volkovskii and L. S. Tsimring, "Synchronization and communication using chaotic frequency modulation," Int. J. Circuit Theory Applicat., vol. 27, pp. 569-576, 1999.

[5] M. Hasler and T. Schimming, "Chaos communication over noisy channels," Int. J. Bifurc. Chaos, vol. 10, pp. 719-735, 2000.

[6] M. Sushchik, L. S. Tsimring, and A. R. Volkovskii, "Performance analysis of correlation-based communication schemes utilizing chaos," IEEE Trans. Circuits Syst. I, vol. 47, pp. 1684-1691, Dec. 2000.

[7] C. K. Tse, K. Y. Cheong, F. C. M. Lau, and S. F. Hau, "Return-mapbased approaches for noncoherent detection in chaotic digital communications," IEEE Trans. Circuits Syst. I, vol. 49, pp. 1495-1499, Oct. 2002.

[8] F. C. M. Lau and C. K. Tse, Chaos-Based Digital Communication Systems. Heidelberg, Germany: Springer-Verlag, 2003.

[9] T. Yang and L. O. Chua, "Chaotic digital code-division multiple access (CDMA) communication systems," Int. J. Bifurc. Chaos, vol. 7, no. 12, pp. 2789-2805, Dec. 1997.

[10] G. Mazzini, G. Setti, and R. Rovatti, "Chaotic complex spreading sequences for asynchronous DS-CDMA. Part I: system modeling and results," IEEE Trans. Circuits Syst. I, vol. 44, pp. 937-947, Oct. 1997.

[11] _ "Chaotic complex spreading sequences for asynchronous DS-CDMA-part II: some theoretical performance bounds," IEEE Trans. Circuits Syst. I, vol. 45, pp. 496-506, Apr. 1998.

[12] G. Kolumbán, M. P. Kennedy, and G. Kis, "Multilevel differential chaos shift keying," in Proc. 5th Int. Specialist Workshop on Nonlinear Dynamics of Electronics Systems (NDES'97), Moscow, Russia, June 1997, pp. 191-196.

[13] Z. Jáko, G. Kis, and G. Kolumbán, "Multiple access capability of the FM-DCSK chaotic communications system," in Proc. 8th Int. Specialist Workshop on Nonlinear Dynamics of Electronics Systems (NDES'2000), Cantania, Italy, May 2000, pp. 52-55.

[14] L. S. Tsimring, A. R. Volkovskii, S. C. Young, and N. F. Rulkov, "Multi-user communication using chaotic frequency modulation," in Proc. Int. Symp. Nonlinear Theory and Its Applications (NOLTA'01), Miyagi, Japan, Oct. 2001, pp. 561-564.

[15] F. C. M. Lau, M. M. Yip, C. K. Tse, and S. F. Hau, "A multiple-access technique for differential chaos shift keying," IEEE Trans. Circuits Syst. I, vol. 49, pp. 96-104, Jan. 2002.

[16] W. M. Tam, F. C. M. Lau, C. K. Tse, and M. M. Yip, "An approach to calculating the bit-error rates of a coherent chaos-shift-keying digital communication system under a noisy multiuser environment," IEEE Trans. Circuits Syst. I, vol. 49, pp. 210-223, Feb. 2002.

[17] G. Kolumbán, M. P. Kennedy, Z. Jákó, and G. Kis, "Chaotic communications with correlator receivers: theory and performance limits," Proc. IEEE, vol. 90, pp. 711-732, May 2002.

[18] F. C. M. Lau, K. Y. Cheong, and C. K. Tse, "Permutation-based DCSK and multiple access DCSK systems," IEEE Trans. Circuits Syst. I, vol. 50, pp. 733-742, June 2003.

[19] J. G. Proakis and M. Salehi, Communications Systems Engineering. Englewood Cliffs, NJ: Prentice-Hall, 1994.

[20] S. Haykin, Adaptive Filter Theory, 3rd ed. Englewood Cliffs, NJ: Prentice-Hall, 1996.

[21] G. Kolumbán and M. P. Kennedy, "The role of synchronization in digital communications using chaos-part III: Performance bounds for correlation receivers," IEEE Trans. Circuits Syst., vol. 47, pp. 1673-1683, Dec. 2000.

[22] W. M. Tam, F. C. M. Lau, and C. K. Tse, "Analysis of bit error rates for multiple access CSK and DCSK communication systems," IEEE Trans. Circuits Syst., vol. 50, pp. 702-707, May 2003. 


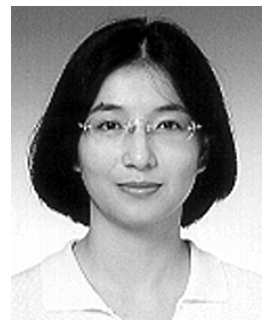

Wai M. Tam received the B.Sc. degree in electronics and information systems from Jinan University, Guangzhou, China, and the M.Phil. and Ph.D. degrees in electronic and information engineering from The Hong Kong Polytechnic University, Hong Kong.

She is currently a Postdoctoral Fellow in the Department of Electronic and Information Engineering, The Hong Kong Polytechnic University, Hong Kong. Her research interests include power control in CDMA mobile cellular systems, third-generation mobile systems, and chaos-based digital communications.

In 2003, she was awarded the Li Po Chun Charitable Trust Fund Scholarship and the Chung Hwa Travel Services Scholarship, and in 2004, The Hong Kong Polytechnic University Postdoctoral Fellowship.

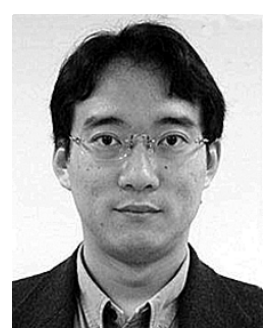

Francis C. M. Lau (M'93-SM'03) received the B.Eng. (Hons.) degree with first class honors in electrical and electronic engineering and the Ph.D. degree from King's College London, University of London, U.K., in 1989 and 1993, respectively.

$\mathrm{He}$ is an Associate Professor and the Leader of the Communication Engineering Section in the Department of Electronic and Information Engineering, The Hong Kong Polytechnic University, Hong Kong. He is the coauthor of Chaos-Based Digital Communication Systems (Heidelberg, Germany: Springer-Verlag, 2003). His main research interests include power control and capacity analysis in mobile communication systems, and chaos-based digital communications.

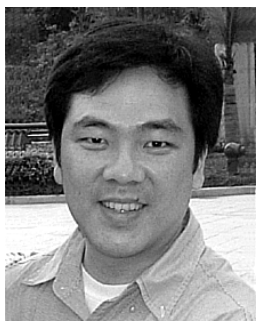

Chi K. Tse (M'90-SM'97) received the B.Eng. (Hons.) degree with first class honors in electrical engineering and the Ph.D. degree from the University of Melbourne, Melbourne, Australia, in 1987 and 1991, respectively.

He is presently a Professor with Hong Kong Polytechnic University, Hong Kong. Since 2002, he has been appointed as Guest Professor by the Southwest China Normal University, Chongqing, China. His research interests include chaotic dynamics, power electronics and chaos-based communications. He is the author of Linear Circuit Analysis (London, U.K.: Addison-Wesley, 1998) and Complex Behavior of Switching Power Converters (Boca Raton, FL: CRC Press, 2003), coauthor of Chaos-Based Digital Communication Systems (Heidelberg, Germany: Springer-Verlag, 2003), and co-holder of a U.S. patent. $\mathrm{He}$ currently serves as an Associate Editor of the International Journal of Systems Science.

In 1987, he was awarded the L.R. East Prize by the Institution of Engineers, Australia, and in 2001, the IEEE TRANSACTIONS ON POWER ELECTRONICS Prize Paper Award. While with the University of Melbourne, he twice received the President's Award for Achievement in Research, the Faculty's Best Researcher Award, and a few other teaching awards. From 1999 to 2001, he served as an Associate Editor of the IEEE TRANSACTIONS ON CIRCUITS AND SYSTEMS PART I-FUNDAMENTAL THEORY AND APPLICATIONS, and since 1999, he has been an Associate Editor for the IEEE TRANSACTIONS ON POWER ELECTRONICS. 